\title{
Youth climate activists meet environmental governance: ageist depictions of the FFF movement and Greta Thunberg in German newspaper coverage
}

\section{Zoe Bergmann \& Ringo Ossewaarde}

To cite this article: Zoe Bergmann \& Ringo Ossewaarde (2020): Youth climate activists meet environmental governance: ageist depictions of the FFF movement and Greta Thunberg in German newspaper coverage, Journal of Multicultural Discourses, DOI: 10.1080/17447143.2020.1745211

To link to this article: https://doi.org/10.1080/17447143.2020.1745211
(C) 2020 The Author(s). Published by Informa UK Limited, trading as Taylor \& Francis Group

曲 Published online: 02 Apr 2020.

\section{Submit your article to this journal $\widetilde{ }$}

\section{山ll Article views: 130}

Q

View related articles

\section{View Crossmark data $₫$}




\section{Youth climate activists meet environmental governance: ageist depictions of the FFF movement and Greta Thunberg in German newspaper coverage}

\section{Zoe Bergmann and Ringo Ossewaarde}

Faculty of Behavioural, Management \& Social Sciences (BMS), Department of Public Administration, University of Twente, Enschede, The Netherlands

\begin{abstract}
In this paper, the domination of the youth climate movement by the use of derogatory ageist language in German newspapers is uncovered. We find that German newspapers use different ageist media images, including 'pupils', 'absentees' and 'dreamers', to delegitimize the FridaysForFuture movement. Greta Thunberg is presented as a 'young hero', who is held responsible for youngsters' absenteeism. FAZ and taz present a paternalist discourse in which the central narrative is that the young climate activists are pupils who are ignorant and still need to learn; and who are obliged by law to go to school. We argue that German newspapers align with the exclusive hegemony of an established environmental governance regime that struggles with the problematic phasing out of coal in Germany. Instead, a common practice of reluctancy and skepticism appears inherited in conservative discussions on climate action led by FAZ and the socalled coal commission.
\end{abstract}

\section{ARTICLE HISTORY}

Received 25 November 2019

Accepted 14 March 2020

\section{KEYWORDS}

Ageism; environmental governance;

FridaysForFuture; Greta Thunberg; media discourse; youth climate activism

\section{Introduction}

Since Greta Thunberg started protesting against climate policies in Sweden in August 2018, public discourse on today's youth engagement in addressing climate change has been traversing the news. Inspired by Thunberg, many young people worldwide have responded and joined climate protests as the Fridays For Future (FFF) movement. On 15 March 2019, more than 1.6 million people from 133 countries took to the street climate action. German youth is accountable for the biggest and most prevalent protests worldwide (Haynes 2019). They have an issue to encounter: the German FFF made its business to protest for a faster and more consequential coal phase out in Germany until 2030 (Fridays For Future Germany [FFF Germany] 2019). Vested interests in German coal are prominent in the discourse on the youth climate movement. The phasing out of coal is a key political challenge in Germany. Stakeholders, including the Commission for Growth,

CONTACT Ringo Ossewaarde m.r.r.ossewaarde@utwente.nl E Faculty of Behavioural, Management \& Social Sciences (BMS), Department of Public Administration, University of Twente, Drienerlolaan 5, 7522NB Enschede, The Netherlands 
Structural Change and Employment (the coal commission) that has been assigned in June 2018 to advice on the phasing out of coal (Federal Ministry for Economic Affairs and Energy [BMWi] 2019), appear to hold on to their vested interests. The widespread coverage of the FFF in German newspapers is embedded in this context of the political challenge of phasing out coal, with longstanding vested interests enacted in ageist depictions of Greta Thunberg and German youth.

While an increasing politicization of youth is recognized (Gencoglu and Yarkin 2019), the ageist domination of climate activist youth through media systems is an underresearched phenomenon. Ageism is typically understood as a phenomenon that older adults experience when being represented in derogatory ways in newspapers, twitter and other media platforms cross countries (Coupland and Coupland 1993; Edström 2018; Fraser, Kenyon, Lagacé, Wittich, and Southall 2016; Gendron et al. 2016; Kroon et al. 2019). Scholars are aware of the risk of discriminatory representations in public media and its potential effect on the 'insiders' and 'outsiders' when it comes to the vulnerable older generation. Ageist media representations of young people have received much less scholarly attention. Some scholars have detected negative media depictions of youth as lacking warmth (Lepianka 2015b) or focussing on crime especially for non-native youth (Lepianka 2015a; Welch, Price, and Yankey 2002). While others have found neutral representations and an increase in quoting youngsters (Levinsen and Wien 2011) or positive portrayals in terms of competence (Lepianka 2015b).

The aim of this paper is to uncover ageist domination of young climate activists in German news media, which reports on youth climate activism in the context of the rulings of the German coal commission. We seek to find out to what extent, and in what ways, German newspaper coverage of the youth climate movement reveals an ageist bias towards young climate activists, in the context of Germany's struggles with vested coal interests. We perform a critical discourse analysis, in the vein of the works of Van Dijk and Wodak, while following Shi-xu $(2016,3)$ notion of abolishing 'the "micro" and the "macro", and reunifies them into one of an all-encompassing and dialectic whole'. In order to account for the political ideologies that are leading the discussion, newspaper articles of the conservative newspaper, FAZ, and the left-wing newspaper, taz, are analysed. This paper aligns with Wodak $(2012,216)$ who argues that, in discourse studies, 'we have thus arrived at the issue of POWER: the power of those who can use language for their various vested interests, (...). Language (and other symbolic systems) is used to determine and define similarities and differences; to draw clear BOUNDARIES between 'us' and 'others".

\section{Ageist domination of youth}

In the past decades, scientific interest in age discrimination has grown rapidly in the context of widespread demographic changes worldwide. Ageism is a concept that was introduced by Butler in 1969 to be able to pinpoint the discrimination of people according to their age. Ageism is primarily understood in terms of prejudices against older people. Butler (1969) defines ageism as a 'deep seated uneasiness of younger generations with ageing' (243). Institutionalized ageist language has a deprecatory, negative character and its terms are derogatory and demeaning on the age group described (Nuessel 1982). Scholars have increasingly pointed at public institutions and media as (re)producers of ageism (Lloyd-Sherlock, Ebrahim, McKee, and Prince 2016; Pritchard-Jones 2017). Media 
shapes an ageist public opinion (Qazi and Shah 2018). Media as such affects how people perceive themselves and 'others'; it constructs categories that include and exclude people (Fraser et al. 2016; Kroon et al. 2019). Age, like religion, ethnicity, class, gender, etc. is one of those identity constructions that gives rise to ingroup and outgroup compositions (othering) - compositions that are based upon similarities and differences. Among other things, the construction of categories like age comes with derogatory language, as outgroups are typically discriminated (Edström 2018; Fraser et al. 2016; Gendron et al. 2016; Kroon et al. 2019). Fraser et al. (2016) expose 13 age-related stereotypes such as being incompetent, vulnerable, a burden on the economy or on others in Canadian print media. They outline the media's creation and maintenance of discursive discriminatory patterns against older adults. Gendron et al. (2016) observe that in social media old age is widely perceived as different (outgroup) while youth is perceived as positive (and ingroup).

Most research on ageism is on the describe discursive domination of older people appears strongly overrepresented. Westman (1991), however, points at the ageist domination of youth, as a systematic neglect of youth's interests. Westman $(1991,240)$ argues that juvenile ageism can be expressed in five different forms, namely when

children are ignored as inherent members of society with development needs as important as the needs and desires of adults; when children are segregated from public places and public media; when parenting is denigrated; when children are treated as adults; and when childcaring systems do not serve the interests of children.

Westman's argument has been widely confirmed, in research on the marginalization and exclusion of young people from public spaces (Moris and Loopmans 2019) and the criminalization of youth (Lepianka 2015a, 2015b; Levinsen and Wien 2011; Welch et al. 2002). Gendron et al. (2016) point at the complexity of ageist language, which includes unintended as well as well-intentioned ageist remarks, ranging from contempt, derogatory expressions to positive images of youth.

When it comes to media coverage on social activism led by young women and teenage girls, positive images may assume the form of a personality cult. Olesen (2016) shows the media construct of young girl heroism in the coverage of Malala Yousafzai. With her activism on education for girls, Malala has become a 'global political and injustice icon' (Olesen 2016, 308). Khurshid and Pitts (2019) emphasize that media coverage of Malala's activism in regard to her being a young girl coincides with the notion that 'adolescent girls have become ideal subjects of neoliberal development and are positioned as vulnerable subjects while also being portrayed as capable of eradicating poverty and global gender inequality' (Khurshid and Pitts 2019, 425). Such positive media constructions, however, may coincide with images of a vulnerable girl, particularly in regard to Malala's Islamic background (Khurshid and Pitts 2019; Qazi and Shah 2018). Malala is no exceptional case of the construction of young female heroism. In the past few years, young female activists have been nominated, and won, the Nobel Peace Prize. Malala Yousafzai was awarded in 2014; Nadia Murad was awarded in 2018. Greta Thunberg was nominated in 2019. Media coverage of youth activism takes place in a particular context of political challenges. For Malala Yousafzai, the context is gender inequality in a patriarchal society. For Nadia Murad, the context is the genocide of Yazidis by ISIL (and the extreme sufferings of Yazidi girls under ISIL rule). For Greta Thunberg, the context is the ecological crisis and the alleged failure of the hegemonic global 
environmental governance regime - a patriarchal regime that enacts vested interests and climate skepticism (Ford 2003).

The context of youth activism is the hegemonic discourse on climate politics that represents the environmental governance regime that is marked by the enactment of climate skepticism (Ford 2003; Gencoglu and Yarkin 2019). Poortinga et al. (2011) find that climate sceptics are typically older individuals, who are politically conservative, hold traditional values and are from lower socio-economic background. Younger individuals, by contrast, tend to cherish self-transcendence and environmental values; and, particularly when they are from a higher socio-economic background, are less commonly climate sceptical (Poortinga et al. 2011). Whitmarsh (2011) adds that climate sceptics are more often men than women. In the German public setting, scholars have identified several climate skeptical German media reports and PR works of public institutions, independent of political ideology (Kaiser and Rhomberg 2016; Rahmstorf 2004). Rhomberg et al (2018) find that in Germany speakers communicate, and are aware of, the necessity of climate action, yet, speakers use the terms target and limit interchangeably, thereby blurring the meaning of the keywords in the discourse.

Our key theoretical assumption is that the news media presents young climate activists in an ageist fashion, so as to reinforce the exclusive hegemony of the established environmental governance regime over the youth climate movement. We expect that mainstream media tends to cover news in support of the status quo, in support of the hegemonic discourse on climate politics (Stegemann and Ossewaarde 2018). We expect that media coverage continues the new trend of portraying young girls as heroes of public activism heroic portraits and positive images that are not devoid of ageist language. In how far climate skepticism functions as interplay in the media portrayals and the policy practice on phasing out of coal in Germany, which embodies the focal point of debate between German climate activists, media and the political scenery, remains to be discovered.

\section{Methods}

We apply critical discourse analysis (CDA) in which we incorporate cultural discourse studies (CDS) as outlined by Shi-xu (2016). CDA is driven by pressing social issues, in which minority groups face prejudices and discriminations (Van Dijk 1993). Through CDA we can 'get more insights into the crucial role of discourse in the reproduction of dominance and inequality' (Van Dijk 1993, 253). CDS is an analytical approach that explores culturally distinctive communication practices in different contexts. CDS allows us to 'abolish the conventional and common binary notions of "text" and "context"' (Shi-xu 2016, 3). In this vein, we perform a (CDA) of two of German newspapers' coverages of youth climate activism, with the deliberate aim of getting more insight into the role of media discourse in the reproduction of ageistdomination of youth (which takes the form of the discursive enactment of media images). CDS enables us explore German newspaper coverage of youth climate activism in the distinctively German context that is marked by the political challenge of phasing out coal.

We analyse German media narratives, which are journalese found in the two German newspapers, the Frankfurter Allgemeine Zeitung (FAZ) and the die tageszeitung (taz) (stylizes itself in small capitals only). Journalese as a term is used to comprehend the jargon of journalists, their dialectic and rhetoric. Both newspapers are so-called 'quality papers', which 
are spread daily, nation-wide, offline as well as online. Due to the increasing use of online sources of information this is of interest. Additionally, both newspapers have the largest reach within their political stance and address a well-educated readership. The conservative $F A Z$ noted addressing more than 850,000 readers each edition and two million visitors on FAZ.net daily (FAZ.NET 2020). The left-wing taz notes 207,000 readers each edition and more than one million unique users on taz.de (taz.de 2017). The study of two of the largest German newspapers with different political stances but similar target audience gives room to the two variables of interest, ageism and its patriarchal connotation, and the progressive youth climate movement, that fights conservative climate policies. The final report on phasing out of coal in Germany from January 2019 complements the chosen dataset of newspaper articles. To research an apparently common practice of climate skepticism that environmental linguistics indicate - which may link ageist newspaper discourse and current climate policy practice in Germany - the linguistic of the abstract on climate protection is in focus.

In performing the CDA, we constructed a coding scheme to discuss the ageist components of the media narratives, due to our deliberate aim of identifying derogatory language. The coding scheme represents theoretical findings on juvenile ageism and linguistic and rhetorical strategies that are identified as helpful in the construction of media representations (Lamb 2013, 344). We selected newspaper articles that portray the FFF movement and Greta Thunberg. The big media debate in Germany newspaper coverage is reflected in the articles we have gathered from 20 August 2018 until 1 May 2019. For this period, the student database WISO (2019b) shows that 16,222 press articles on the keywords FFF and Greta Thunberg have been released in newspapers worldwide. The database is free-to-use for academic staff and students of German universities. The German press is accountable for 13,355 of all these articles (WISO 2019a). In our initial attempts, we obtained, from the database WISO and the FAZ Archive, 228 articles from FAZ and 162 articles from taz. The choice to study two divers' newspapers only allowed us to check all of these 390 articles on content. We excluded articles under 100 words; we took them as too empty in substance for an analysis of discursive and dialectic elements. We also excluded letters to the editor or opinions by the public, as this is no journalese of the newspaper. We checked the content of the articles on reports that specifically discuss the FFF or Greta Thunberg. The pre-selection resulted in 71 articles from taz and 100 articles from FAZ. The articles were selected in regard to their title, for which three factors have been decisive. The title had to either directly or indirectly refer to the names Greta Thunberg or FFF, their climate protest, or their young age. The final selection includes 39 articles of taz and 59 of FAZ, leading to a total of 98 newspaper articles that were analysed in all detail. We present few rhetorical devices and linguistics as well as one longer excerpts of both newspaper's discourses in the next section, in order to justify and illustrate our argument. As the newspaper articles are originally published in the German language, excerpts have been translated by ourselves into English.

\section{Media images of the FridaysForFuture and Greta Thunberg}

Phasing-out coal is the key issue of German environmental governance and the youth climate activists. We retrace four ageist media images - pupil, absentee, dreamer, young heroine - that FAZ and taz discursively construct, in the context of the political 
struggle of phasing out coal in Germany. Vested interests become explicit in the ageist language used, with German newspapers acting as a hegemon Tables 1-3.

\section{The pupils}

The most striking media image that both FAZ and taz create throughout the discourse is the portrayal of the global FFF movement as a pupil-protest, with youth climate activists being presented as pupils (in 85 articles of the 98 in total). Both newspapers make great use of rhetorical devices like 'A great advanced course in protest culture' (taz, 56) or 'On the road instead of the classroom' (FAZ, 163) and argumentative strategies have been used in order to portray the youth climate activism in accord with their position as pupils. Being constructed as pupils, young climate activists are portrayed as inherently different. This categorizes the FFF as an outgroup, which is deleted as an actual actor from its sentences, strengthening its image of the domesticated pupil. How both newspapers engage in these strategies can be observed in the examples given on pages 11 and 12. The climate activists or the climate movement are removed from the sentences, and they are depicted according to the 'pupil-being'. The use of this nomination directs the focus of the debate on their function as 'pupils', who are in the process of learning, instead of depicting them as what they are essentially doing in the situation, that is, protesting against the failure of the established environmental governance regime to address climate change and to phase out fossil energy, particularly coal, as quickly as possible. It diverts the debate from climate activism on to the youthfulness of the activists, that they are not yet fully educated, and neglects what the youth climate movement essentially aims and stands for, which is first of all climate action. Children's interests are disregarded by both newspapers' coverages. Furthermore, the 'pupil'-image promotes subordination

Table 1. Coding scheme to study the use of ageist language.

\begin{tabular}{|c|c|c|c|}
\hline Text analysis & Theoretical concepts & Code & Exemplary keywords \\
\hline \multirow[t]{9}{*}{ Content } & \multirow{5}{*}{$\begin{array}{l}\text { Institutional juvenile } \\
\text { ageism }\end{array}$} & Youth is subordinated & Inferior, uninformed, inexperienced \\
\hline & & Youth does not belong in public & Out of place, illegitimate, misguidec \\
\hline & & Parenting is a burden & Exhausting, stressful, demanding \\
\hline & & Youth is treated as adults & Women, men, immature \\
\hline & & Youth's interests are minor & Unimportant, unrealistic, irrelevant \\
\hline & Derogatory language & $\begin{array}{l}\text { Youngsters have negative } \\
\text { characteristics }\end{array}$ & Childish, naive, bad \\
\hline & \multirow{3}{*}{$\begin{array}{l}\text { Outgroup } \\
\text { discrimination }\end{array}$} & Youth is different & They $\neq$ we, the children, youth \\
\hline & & Being young is negative & Too young, only ... aged, childish \\
\hline & & Being old is positive & Wise, manner, knowledgeable \\
\hline \multirow{7}{*}{$\begin{array}{l}\text { Linguistic and } \\
\text { rhetorical } \\
\text { strategies }\end{array}$} & Predication & $\begin{array}{l}\text { Young people do have certain } \\
\text { characteristics }\end{array}$ & To be explored \\
\hline & \multirow[t]{5}{*}{ Nomination } & $\begin{array}{l}\text { Functionalization (actor named as what } \\
\text { he/she/it does) }\end{array}$ & To be explored \\
\hline & & $\begin{array}{l}\text { Abstraction (actor named as the } \\
\text { 'problem' may related to the } \\
\text { her/him/it) }\end{array}$ & To be explored \\
\hline & & $\begin{array}{l}\text { Spatialization (actor named as what } \\
\text { place may be referred to it/her/him) }\end{array}$ & To be explored \\
\hline & & $\begin{array}{l}\text { Utterance autonomization (actor } \\
\text { named as what he/she/it claims) }\end{array}$ & To be explored \\
\hline & & $\begin{array}{l}\text { Instrumentalization (actor named as a } \\
\text { mechanism applicable to her/him/it) }\end{array}$ & To be explored \\
\hline & Rhetorical elements & metaphors, hyperboles, comparisons & To be explored \\
\hline
\end{tabular}


Table 2. Media images of the youth climate movement and Greta Thunberg.

\begin{tabular}{|c|c|c|c|}
\hline Media image & $\begin{array}{l}\text { Total } \\
\text { Code frequency// article } \\
\text { count }\end{array}$ & $\begin{array}{c}\text { Frankfurter Allgemeine } \\
\text { Zeitung } \\
\text { Code frequency// article } \\
\text { count }\end{array}$ & $\begin{array}{c}\text { die tageszeitung } \\
\text { Code frequency// article } \\
\text { count }\end{array}$ \\
\hline $\begin{array}{l}\text { The pupils } \\
\text { pupils } \\
\text { pupil strike }\end{array}$ & $383 / /$ in 85 articles & $236 / /$ in 55 articles & $147 / /$ in 30 articles \\
\hline $\begin{array}{l}\text { The absentees } \\
\text { compulsory education law } \\
\text { Skip school }\end{array}$ & $64 / /$ in 29 articles & 44// in 19 articles & $20 / /$ in 10 articles \\
\hline $\begin{array}{l}\text { The dreamers } \\
\text { unwarranted claims } \\
\text { unrealistic claims } \\
\text { not well thought } \\
\text { (too) young }\end{array}$ & $48 / /$ in 21 articles & $38 / /$ in 14 articles & $8 / /$ in 7 articles \\
\hline $\begin{array}{l}\text { The personality cult of Greta } \\
\text { Thunberg } \\
\text { The hero } \\
\text { Young girl }\end{array}$ & $175 / /$ in 71 articles & $100 / /$ in 43 articles & $75 / /$ in 28 articles \\
\hline
\end{tabular}

of the young group, that is being active, and creates an inferior position for them based on their alleged ignorance (they still need to go to school). Likewise, the pupil image enforces what Westman (1991) calls children's segregation from public spaces: newspapers signal that children are pupils who are to be found at school.

\section{The absentees}

In FAZ and taz media narratives, journalists portray the young climate activists in terms of skipping school, which makes their activism a form of absenteeism (in 29 articles of the total of 98). In the construction of the image of the absentee, FAZ and taz frequently refer to the compulsory education law. To question whether it is legitimate to violate the education law (skipping school) for purpose of protest, mildly criminalize the youth climate activists. How both newspapers engage in this image creation becomes visible in the examples given later (11-12). In addition, the global FFF movement is represented as absentee through the strategic use of abstract expressions and rhetorical devices, such as 'Absenteeism for Future' (FAZ, 155). As in the image of the pupil, also the absentee construct locates the young climate activist in school rather than in public space. And as with the image of the pupil, the image of the absentee as well directs the focus and the issue of debate away from the actual interests of the youth movement. Within this construction, the interests of the young climate activists are disregarded - and their interests do not equal the interests of the public system that aims for education of the young. Due to the immanent references to the breaching of the law and the outlining of the 'illegal protests', the coverage reinforcement of the absentee image is ageist, as it segregates youth from public places. The absentee image delegitimizes the public protests and argues that pupils are not eligible for the basic human right to demonstrate when they want to. With that enactment, young people are constructed as an outgroup, a different group that have negative (law-breaking) characteristics. Here, this image does not only promote 'othering', but it also facilitates the picture of a criminal 'other' who upsets order. The image of the absentee facilitates negative perceptions of the newspaper readers on the youth climate movement, as the focus is directed on an indecent youth. Such framing is 
Table 3. Data collection and dataset.

\begin{tabular}{|c|c|c|c|}
\hline No. & Newspaper & Article (title and weblink) & $\begin{array}{c}\text { (Pre- } \\
\text { )selected }\end{array}$ \\
\hline 1 & taz & Erwachsene haben nicht so viel Zukunft & \\
\hline \multirow[t]{2}{*}{2} & & Italiens Jugend gibt Gretas Mission starken Linksdrall & $\mathrm{x}$ \\
\hline & & https://www.wiso-net.de/document/TAZ_T20192004.5587005/hitlist/0?all= & \\
\hline 3 & & Sie haben es nicht verkackt! & \\
\hline 4 & & Das ist regenerative Kultur & \\
\hline 5 & & Auf sie mit Gebrüll & \\
\hline 6 & & Das Dilemma der zukünftigen Weltentdecker & \\
\hline \multirow[t]{2}{*}{7} & & Future für wen? Nicht nur für die Elite & $\mathrm{x}$ \\
\hline & & https://www.wiso-net.de/document/TAZ_T20191804.5589135/hitlist/0?all= & \\
\hline \multirow[t]{2}{*}{8} & & Die Alten lassen die Jungen im Stich & $\mathrm{x}$ \\
\hline & & https://www.wiso-net.de/document/TAZ__T20191804.5586820/hitlist/25?all= & \\
\hline \multirow[t]{2}{*}{9} & & Aufstand ums Klima & $\mathrm{x}$ \\
\hline & & https://www.wiso-net.de/document/TAZ_T20191804.5586821/hitlist/25?all= & \\
\hline \multirow[t]{2}{*}{10} & & Mit Greta, aber radikaler & $\mathrm{x}$ \\
\hline & & https://www.wiso-net.de/document/TAZ__T20191604.5588500/hitlist/25?all= & \\
\hline \multirow[t]{2}{*}{11} & & Greta, Jesus und die Klimakatastrophe & $\mathrm{x}$ \\
\hline & & https://www.wiso-net.de/document/TAZ_T20191504.5584963/hitlist/25?all= & \\
\hline 12 & & Die fetten Jahre sind vorbei & \\
\hline \multirow[t]{2}{*}{13} & & Es war wieder Friday & $x$ \\
\hline & & https://www.wiso-net.de/document/TAZ__T20191304.5587444/hitlist/25?all= & \\
\hline \multirow[t]{2}{*}{14} & & Being Greta Thunberg & $\mathrm{x}$ \\
\hline & & https://www.wiso-net.de/document/TAZ__T20191204.5584889/hitlist/25?all= & \\
\hline 15 & & Esther Slevogt betrachtet das Treiben auf Berlins Bühnen & \\
\hline \multirow[t]{2}{*}{16} & & Future ohne Fridays? & $\mathrm{x}$ \\
\hline & & https://www.wiso-net.de/document/TAZ_T20191004.5583928/hitlist/25?all= & \\
\hline \multirow[t]{2}{*}{17} & & Komplimente reichen Aktivist*innen nicht & $x$ \\
\hline & & https://www.wiso-net.de/document/TAZ_T20190904.5583819/hitlist/50?all= & \\
\hline 18 & & Wir müssen eben Prioritäten setzen & \\
\hline 19 & & Streik mit Teach-in & \\
\hline 20 & & Verzichten wir - auf diese Regierung & \\
\hline 21 & & Dumbledore wouldn't let this happen & \\
\hline \multirow[t]{2}{*}{22} & & Berlin feiert Greta & $\mathrm{x}$ \\
\hline & & https://www.wiso-net.de/document/TAZ__T20193003.5581752/hitlist/50?all= & \\
\hline \multirow[t]{2}{*}{23} & & Jeder kann Greta sein & $\mathrm{x}$ \\
\hline & & https://www.wiso-net.de/document/TAZ__T20193003.5581880/hitlist/50?all= & \\
\hline 24 & & Fünf Fragen zur Zukunft & \\
\hline 25 & & Größer als die 68er & \\
\hline \multirow[t]{2}{*}{26} & & Gute Nachricht wäre: \#WiralleforFuture & $\mathrm{x}$ \\
\hline & & https://www.wiso-net.de/document/TAZ__T20192903.5584044/hitlist/50?all= & \\
\hline \multirow[t]{2}{*}{27} & & Schlaue linke Greta-Fans & $x$ \\
\hline & & https://www.wiso-net.de/document/TAZ_T20192803.5581517/hitlist/50?all= & \\
\hline \multirow[t]{2}{*}{28} & & Lieber Schule schwänzen als nichts fürs Klima tun! & $\mathrm{x}$ \\
\hline & & https://www.wiso-net.de/document/TAZ__T20192703.5582939/hitlist/50?all= & \\
\hline 29 & & Eltern wollen keine Sanktionen & \\
\hline \multirow[t]{2}{*}{30} & & Tausend fürs Klima & $x$ \\
\hline & & https://www.wiso-net.de/document/TAZ__T20192303.5582552/hitlist/75?all= & \\
\hline 31 & & Fieses Schüler-Dilemma & \\
\hline \multirow[t]{2}{*}{32} & & Thunberg kommt nach Berlin & $\mathrm{x}$ \\
\hline & & https://www.wiso-net.de/document/TAZ__T20192003.5575635/hitlist/75?all= & \\
\hline 33 & & Das ist politische Feigheit vor der Sympathiewelle & \\
\hline 34 & & Angekettet for future & $x$ \\
\hline & & https://www.wiso-net.de/document/TAZ_T20191903.5578654/hitlist/75?all= & \\
\hline 35 & & Greta und Barbie & $\mathrm{x}$ \\
\hline & & https://www.wiso-net.de/document/TAZ__T20191803.5578527/hitlist/75?all= & \\
\hline 36 & & Anders als die anderen & \\
\hline 37 & & Radikal angepasst & \\
\hline 38 & & Große Geste in kleinen Städten & \\
\hline 39 & & Fünfmal mehr als erwartet & \\
\hline 40 & & Wie kommen wir uns näher? & \\
\hline 41 & & & $\mathrm{x}$ \\
\hline
\end{tabular}


Table 3. Continued.

\begin{tabular}{|c|c|c|c|}
\hline No. & Newspaper & Article (title and weblink) & $\begin{array}{c}\text { (Pre- } \\
\text { )selected }\end{array}$ \\
\hline & & $\begin{array}{l}\text { Make the World Greta again } \\
\text { https://www.wiso-net.de/document/TAZ_T20191603.5577796/hitlist/75?all= }\end{array}$ & \\
\hline 42 & & Panik können wir uns nicht leisten & \\
\hline 43 & & $\begin{array}{l}\text { Nehmt Greta Thunberg Ernst } \\
\text { https://www.wiso-net.de/document/TAZ_T20191603.5577705/hitlist/75?all= }\end{array}$ & $\mathrm{x}$ \\
\hline 44 & & Weltretter mit Hasenherz & \\
\hline 45 & & Kostümierte Kackhaufen & \\
\hline 46 & & Wir brauchen die, die jetzt im Bundestag sitzen & \\
\hline 47 & & $\begin{array}{l}\text { Sie sind jung und sie brauchen die Welt } \\
\text { https://www.wiso-net.de/document/TAZ_T20191403.5577934/hitlist/100?all= }\end{array}$ & $\mathrm{x}$ \\
\hline 48 & & $\begin{array}{l}\text { Proteste weltweit am Friday for Future } \\
\text { https://www.wiso-net.de/document/TAZ_T20191403.5578106/hitlist/100?all= }\end{array}$ & $\mathrm{x}$ \\
\hline 49 & & $\begin{array}{l}\text { Greta global } \\
\text { https://www.wiso-net.de/document/TAZ_T20191403.5578109/hitlist/100?all= }\end{array}$ & $\mathrm{x}$ \\
\hline 50 & & $\begin{array}{l}\text { Schulstreik begeistert Hochschullehrerlnnen } \\
\text { https://www.wiso-net.de/document/TAZ_T20190603.5578232/hitlist/100?all= }\end{array}$ & $\mathrm{x}$ \\
\hline 51 & & $\begin{array}{l}\text { Greta Thunberg - der Star, der keiner sein will } \\
\text { https://www.wiso-net.de/document/TAZ_T20190203.5574019/hitlist/100?all= }\end{array}$ & $\mathrm{x}$ \\
\hline 52 & & $\begin{array}{l}\text { Tausende streiken mit Greta } \\
\text { https://www.wiso-net.de/document/TAZ_T20190203.5574064/hitlist/100?all= }\end{array}$ & $\mathrm{x}$ \\
\hline 53 & & $\begin{array}{l}\text { Mit Greta und den Eltern } \\
\text { https://www.wiso-net.de/document/TAZ_T20190103.5574432/hitlist/100?all= }\end{array}$ & $\mathrm{x}$ \\
\hline 54 & & Ganz normal dabei & \\
\hline 55 & & $\begin{array}{l}\text { Warum kritisieren wir Kinder, die für den Erhalt des Planeten auf die Straße gehen? } \\
\text { https://www.wiso-net.de/document/TAZ_T20192702.5572517/hitlist/100?all= }\end{array}$ & $\mathrm{x}$ \\
\hline 56 & & $\begin{array}{l}\text { Ein prima Leistungskurs in Protestkultur } \\
\text { https://www.wiso-net.de/document/TAZ_T20192302.5572897/hitlist/125?all= }\end{array}$ & $\mathrm{x}$ \\
\hline 57 & & $\begin{array}{l}\text { Wunderbarer Freitag } \\
\text { https://www.wiso-net.de/document/TAZ_T20192302.5572925/hitlist/125?all= }\end{array}$ & $\mathrm{x}$ \\
\hline 58 & & Politischer Einsatz? Passt PolitikerInnen eher gar nicht & \\
\hline 59 & & Lernziel verfehlt & \\
\hline 60 & & $\begin{array}{l}\text { Hüpfen gegen die Kohle } \\
\text { https://www.wiso-net.de/document/TAZ_T20191502.5570360/hitlist/125?all= }\end{array}$ & $\mathrm{x}$ \\
\hline 61 & & $\begin{array}{l}\text { Achtung, hier kommt mein Kind! } \\
\text { https://www.wiso-net.de/document/TAZ_T20191302.5569275/hitlist/125?all= }\end{array}$ & $\mathrm{x}$ \\
\hline 62 & & $\begin{array}{l}\text { Fridays for Future: Druck von oben } \\
\text { https://www.wiso-net.de/document/TAZ_T20190102.5569721/hitlist/125?all= }\end{array}$ & $\mathrm{x}$ \\
\hline 63 & & Torben Becker sichtet die sozialen Bewegungen der Stadt & \\
\hline 64 & & Hass und Hetze & \\
\hline 65 & & Mehr Druck ist hilfreich & \\
\hline 66 & & $\begin{array}{l}\text { Demo fürs Klima } \\
\text { https://www.wiso-net.de/document/TAZ_T20192601.5568204/hitlist/150?all= }\end{array}$ & $\mathrm{x}$ \\
\hline 67 & & $\begin{array}{l}\text { Irgendjemand muss das Klima ja retten } \\
\text { https://www.wiso-net.de/document/TAZ_T20192401.5565304/hitlist/150?all= }\end{array}$ & $\mathrm{x}$ \\
\hline 68 & & $\begin{array}{l}\text { 25,000 Schülerlnnen streiken fürs Klima } \\
\text { https://www.wiso-net.de/document/TAZ_T20191901.5564031/hitlist/150?all= }\end{array}$ & $\mathrm{x}$ \\
\hline 69 & & Endspiel um das Abkommen & \\
\hline 70 & & $\begin{array}{l}\text { Klimastreik bewegt die Welt } \\
\text { https://www.wiso-net.de/document/TAZ_T20180312.5553074/hitlist/150?all= }\end{array}$ & $x$ \\
\hline 71 & & $\begin{array}{l}\text { Erst 15, aber knallhart: Die Schülerin Greta Thunberg streikt in Stockholm fürs Klima } \\
\text { https://www.wiso-net.de/document/TAZ_T20182708.5528023/hitlist/150?all= }\end{array}$ & $x$ \\
\hline 72 & FAZ & Selbstherrlich & \\
\hline 73 & & Die Welt ist gar nicht so übel & \\
\hline 74 & & Müllberg auf dem Römerberg & \\
\hline 75 & & Die Revolution auf dem Teller & \\
\hline 76 & & Der Unrat anderer Leute eingetütet & \\
\hline 77 & & $\begin{array}{l}\text { Tausende protestieren in Rom mit Greta Thunberg } \\
\text { https://www.faz-biblionet.de/faz-portal/document?uid=FAZN_20190419_- } \\
\text { 6148763\&token=598ddf2e-c00d-4015-8586-4b227be80c1d\&p._scr=faz-archiv\&p.q= } \\
\text { greta+thunberg+oder+fridays+for+future\&p.source=\&p.max=10\&p.sort=\&p.offset= }\end{array}$ & $x$ \\
\hline
\end{tabular}


Table 3. Continued.

\begin{tabular}{|c|c|c|c|}
\hline No. & Newspaper & Article (title and weblink) & $\begin{array}{c}\text { (Pre- } \\
\text { )selected }\end{array}$ \\
\hline & & $\begin{array}{l}\text { 30\&p._ts=1562069746307\&p.DT_from=20.08.2018\&p.DT_to=01.05.2019\&p. } \\
\text { timeFilterType }=0\end{array}$ & \\
\hline 78 & & Schüler sammeln freiwillig Müll auf Frankfurts Straßen & \\
\hline 79 & & Er lächelte und lachte die ganze Zeit & \\
\hline 80 & & Andere Städte - Gleiche Nöte & \\
\hline 81 & & $\begin{array}{l}\text { Greta Thunberg trifft auf Papst Franziskus } \\
\text { https://www.faz-biblionet.de/faz-portal/document?uid=FAZN_20190415_- } \\
\text { 6141982\&token=562f479a-2abf-42ca-a968-d36fb5ef331f\&p._scr=faz-archiv\&p.q=greta } \\
\text { +thunbeg+oder+fridays+for+future\&p.source=\&p.max=10\&p.sort=\&p.offset=20\&p. } \\
\text { ts=1562069864563\&p.DT_from=20.08.2018\&p.DT_to=01.05.2019\&p.timeFilterType=0 }\end{array}$ & $\mathrm{x}$ \\
\hline 82 & & $\begin{array}{l}\text { Demonstrieren nur außerhalb der Unterrichtszeit } \\
\text { https://www.faz-biblionet.de/faz-portal/document?uid=RMO_- } \\
\text { FDA201904135686076\&token=e2c3544b-5173-4475-8974-6a04b1a38b0e\&p._scr=faz- } \\
\text { archiv\&p.q=greta+thunbeg+oder+fridays+for+future\&p.source=\&p.max=10\&p.sort= } \\
\text { \&p.offset=30\&p._ts=1562069899426\&p.DT_from=20.08.2018\&p.DT_to=01.05.2019\&p. } \\
\text { timeFilterType=0 }\end{array}$ & $\mathrm{x}$ \\
\hline 83 & & Wie viel kostet es mich, das Klima zu retten? & \\
\hline 84 & & Unser Abdruck & \\
\hline 85 & & $\begin{array}{l}\text { Bauträger Instone bietet Schülern von "Fridays for Future" Gespräch an } \\
\text { https://www.faz-biblionet.de/faz-portal/document?uid=RMO_- } \\
\text { FDA201904125685052\&token=eb15bef3-7a93-4ae5-ada1-715c237d3647\&p._scr=faz- } \\
\text { archiv\&p.q=greta+thunbeg+oder+fridays+for+future\&p.source=\&p.max=10\&p.sort= } \\
\text { \&p.offset=30\&p._ts=1562069926616\&p.DT_from=20.08.2018\&p.DT_to=01.05.2019\&p. } \\
\text { timeFilterType=0 }\end{array}$ & $\mathrm{x}$ \\
\hline 86 & & $\begin{array}{l}\text { Hessens Kultusminister kritisiert Schulausfall durch Klimademos } \\
\text { https://www.faz-biblionet.de/faz-portal/document?uid=FAZN_20190412__ } \\
\text { 6137866\&token=efee8cc7-7024-407a-b8e9-4969759bc94a\&p._scr=faz-archiv\&p.q=greta } \\
\text { +thunbeg+oder+fridays+for+future\&p.source=\&p.max=10\&p.sort=\&p.offset=30\&p._ } \\
\text { ts=1562069943059\&p.DT_from=20.08.2018\&p.DT_to=01.05.2019\&p.timeFilterType=0 }\end{array}$ & $\mathrm{x}$ \\
\hline 87 & & Protestierer mahnen Luftfahrt zur Eile & \\
\hline 88 & & $\begin{array}{l}\text { Gretas Protest überfordert uns } \\
\text { https://www.faz-biblionet.de/faz-portal/document?uid=FAZN_20190411__ } \\
\text { 6136616\&token=21a2cc4c-aa3e-408e-bc44-0b3dc6fdbb09\&p._scr=faz-archiv\&p.q=greta } \\
\text { +thunbeg+oder+fridays+for+future\&p.source=\&p.max=10\&p.sort=\&p.offset=30\&p._ } \\
\text { ts=1562069961295\&p.DT_from=20.08.2018\&p.DT_to=01.05.2019\&p.timeFilterType=0 }\end{array}$ & $x$ \\
\hline 89 & & $\begin{array}{l}\text { Schüler fordern Nettonull bis } 2035 \\
\text { https://www.faz-biblionet.de/faz-portal/document?uid=FAZ } \\
\text { FD2201904095681769\&token=d5675b9b-b1ed-4528-a4ae-85376a0e9013\&p._scr=faz- } \\
\text { archiv\&p.q=greta+thunbeg+oder+fridays+for+future\&p.source=\&p.max=10\&p.sort= } \\
\text { \&p.offset=40\&p._ts=1562069982997\&p.DT_from=20.08.2018\&p.DT_to=01.05.2019\&p. } \\
\text { timeFilterType=0 }\end{array}$ & $x$ \\
\hline 90 & & $\begin{array}{l}\text { CDU sucht Gespräch mit Schülerdemonstranten } \\
\text { https://www.faz-biblionet.de/faz-portal/document?uid=FAZ } \\
\text { FD2201904095682058\&token=de19bdae-09c3-4f82-86bc-267b0c82fc95\&p._scr=faz- } \\
\text { archiv\&p.q=greta+thunbeg+oder+fridays+for+future\&p.source=\&p.max=10\&p.sort= } \\
\text { \&p.offset=40\&p._ts=1562069995479\&p.DT_from=20.08.2018\&p.DT_to=01.05.2019\&p. } \\
\text { timeFilterType=0 }\end{array}$ & $\mathrm{x}$ \\
\hline 91 & & Eine Warnung aus dem Dinosaurier-Saal & \\
\hline 92 & & $\begin{array}{l}\text { Das sind die Forderungen der "Fridays for Future"-Demonstranten } \\
\text { https://www.faz-biblionet.de/faz-portal/document?uid=FAZN_20190408__ } \\
\text { 6130706\&token=f216d8cd-0c0d-4413-921e-43e4d68fb45b\&p._scr=faz-archiv\&p.q= } \\
\text { greta+thunbeg+oder+fridays+for+future\&p.source=\&p.max=10\&p.sort=\&p.offset= } \\
\text { 40\&p._ts=1562070011158\&p.DT_from=20.08.2018\&p.DT_to=01.05.2019\&p. } \\
\text { timeFilterType=0 }\end{array}$ & $\mathrm{x}$ \\
\hline 93 & & $\begin{array}{l}\text { Schüler demonstrieren für "Grüne Lunge" } \\
\text { https://www.faz-biblionet.de/faz-portal/document?uid=RMO_- } \\
\text { FDA201904065680630\&token=bf8895ea-372d-41dd-86d9-454fa1dd01ce\&p._scr=faz- } \\
\text { archiv\&p.q=greta+thunbeg+oder+fridays+for+future\&p.source=\&p.max=10\&p.sort= } \\
\text { \&p.offset=40\&p._ts=1562070029400\&p.DT_from=20.08.2018\&p.DT_to=01.05.2019\&p. } \\
\text { timeFilterType=0 }\end{array}$ & $\mathrm{x}$ \\
\hline
\end{tabular}


Table 3. Continued.

\begin{tabular}{lcc}
\hline No. Newspaper & Article (title and weblink) & $\begin{array}{c}\text { (Pre- } \\
\text { )selected }\end{array}$ \\
\hline 94 & Ich bin fassungslos, das Schulschwänzen heiliggesprochen wird & x
\end{tabular}

https://www.faz-biblionet.de/faz-portal/document?uid=FAZN_20190406_

6127432\&token=441db09b-ec21-41a3-9d2a-3848818cf46b\&p._scr=faz-archiv\&p.q= greta+thunbeg+oder+fridays+for+future\&p.source=\&p.max=10\&p.sort=\&p.offset= 40\&p._ts=1562070045331\&p.DT_from=20.08.2018\&p.DT_to=01.05.2019\&p. timeFilterType $=0$ archiv\&p.q=greta+thunbeg+oder+fridays+for+future\&p.source=\&p.max=10\&p.sort= \&p.offset=40\&p._ts=1562070062794\&p.DT_from=20.08.2018\&p.DT_to=01.05.2019\&p. timeFilterType $=0$ +thunbeg+oder+fridays+for+future\&p.source $=\&$ p. $m a x=10 \& p . s o r t=\& p . o f f s e t=50 \& p$. $\mathrm{ts}=1562070121057 \&$ p.DT_from $=20.08 .2018 \&$ p.DT_to $=01.05 .2019 \&$ p.timeFilterType $=0$ We will rock you archiv\&p.q=greta+thunbeg+oder+fridays+for+future \&p.source $=\& p . \max =10 \& p$. sort $=$ \&p.offset $=50 \&$ p._ts $=1562070139416 \&$ p.DT_from $=20.08 .2018 \&$ p.DT_to $=01.05 .2019 \&$ p. timeFilterType $=0$ https://www.faz-biblionet.de/faz-portal/document?uid=FAZN_20190330

6116717\&token=f5b41e77-fed9-4bc8-8666-9a20f5077545\&p._scr=faz-archiv\&p.q=greta +thunbeg+oder+fridays+for+future\&p.source $=\&$ p.max $=10 \&$ p.sort $=\&$ p.offset $=60 \&$ p. ts $=1562070157611 \&$ p.DT_from $=20.08 .2018 \&$ p.DT_to $=01.05 .2019 \&$ p.timeFilterType $=0$ Wir stehen an einem Scheideweg unserer Geschichte Greta ist die Beste https://www.faz-biblionet.de/faz-portal/document?uid=FAZN__20190329_ 6114861\&token=c643196a-f878-4c22-a919-bf5962458efd\&p._scr=faz-archiv\&p.q=greta +thunbeg+oder+fridays+for+future\&p.source $=\&$ p.max $=10 \&$ p.sort $=\& p . o f f s e t=60 \& p$. ts $=1562070171959 \&$ p.DT_from $=20.08 .2018 \& p . D T \_t o=01.05 .2019 \& p$. timeFilterType $=0$ In einer Krise muss man sein Verhalten ändern Mit Kabarettisten doof denken Klimastreik bei Volkswagen https://www.faz-biblionet.de/faz-portal/document?uid=FAZN__20190323_ 6104735\&token=1976e0ef-fab3-4e9e-95c0-e638c527d698\&p._scr=faz-archiv\&p.q=greta +thunbeg+oder+fridays+for+future\&p.source $=\&$ p.max $=10 \&$ p.sort $=\& p . o f f s e t=60 \& p$. ts $=1562070211441 \&$ p.DT_from $=20.08 .2018 \&$ p.DT_to $=01.05 .2019 \&$ p.timeFilterType $=0$ Mehr Daniel Düsentrieb wagen

Der Greta-Effekt https://www.faz-biblionet.de/faz-portal/document?uid=FAZN 20190322_6103010\&token=de55c1ac-f28c-420c-b8a2-26c0f1bcbe1b\&p._scr=fazarchiv\&p.q=greta+thunbeg+oder+fridays+for+future\&p.source=\&p.max=10\&p.sort= \&p.offset=70\&p._ts=1562070232259\&p.DT_from $=20.08 .2018 \&$ p.DT_to=01.05.2019\&p. timeFilterType $=0$ Note 6 warum+greta+thunberg+atomlobbyismus\&p.source=\&p.max=10\&p.sort=\&p.offset $=$ 0\&p._ts=1562070741393\&p.DT_from=20.08.2018\&p.DT_to=01.05.2019\&p.

timeFilterType $=0$ 
Table 3. Continued.

\begin{tabular}{lrr}
\hline & & (Pre- \\
No. Newspaper & Article (title and weblink) & )selected
\end{tabular}

archiv\&p.q=greta+thunbeg+oder+fridays + for + future $\&$ p.source $=\& p \cdot \max =10 \& p \cdot$.sort $=$ \&p.offset $=70 \& p . \_t s=1562070305351 \&$ p.DT_from $=20.08 .2018 \&$ p.DT_to $=01.05 .2019 \&$ p. timeFilterType $=0$

Meine Familie fliegt schon gerne in den Urlaub

Am Freitagmorgen wird die Welt gerettet

https://www.faz-biblionet.de/faz-portal/document?uid=RMO

FDA201903165662757\&token=d011a5b1-5e2e-463a-8913-e43acdac6ec6\&p._scr=fazarchiv\&p.q=greta+thunbeg+oder+fridays+for+future\&p.source $=\& p . \max =10 \& p$. sort $=$ \&p.offset=80\&p._ts=1562070339416\&p.DT_from $=20.08 .2018 \&$ p.DT_to=01.05.2019\&p. timeFilterType $=0$

Wie tickt Greta Thunberg? https://www.faz-biblionet.de/faz-portal/document?uid=FAZN 20190316_6093170\&token=961942a3-c916-4fec-a245-a8df81418f88\&p._scr=faz-

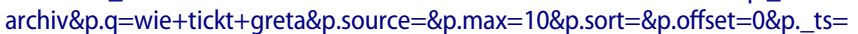
1562070777114\&p.DT_from $=20.08 .2018 \&$ p.DT_to $=01.05 .2019 \&$ p.timeFilterType $=0$ Die Europäische Union soll bis 2050 klimaneutral sein Schulämter fordern Sanktionen Die Profis sind da Die Schülerdemos stellen den Kohlekompromiss infrage https://www.faz-biblionet.de/faz-portal/document?uid=FAZN__20190315_ 6091548\&token=41566f1c-f294-459d-9e84-104cd10fed1b\&p._scr=faz-archiv\&p.q=greta +thunbeg+oder+fridays+for+future\&p.source $=\&$ p. $m a x=10 \&$ p.sort $=\& p$. offset $=80 \& p$. ts $=1562070382193 \& p . D T \_$from $=20.08 .2018 \& p . D T \_t o=01.05 .2019 \& p . t i m e F i l t e r T y p e=0$ Greta inspiriert dazu, es ihr gleichzutun https://www.faz-biblionet.de/faz-portal/document?uid=FAZN__20190315_ 6088880\&token=20640a20-8f16-4dd4-a3ae-f39f17c870b7\&p._scr=faz-archiv\&p.q=greta +inspiriert\&p.source $=\& p . \max =10 \& p . s o r t=\& p . o f f s e t=0 \& p .+t s=1562070700839 \& p . D T$ from $=20.08 .2018 \&$ p.DT_to $=01.05 .2019 \&$ p.timeFilterType $=0$

Schüler in mehr als 100 Staaten demonstrieren für mehr Klimaschutz https://www.faz-biblionet.de/faz-portal/document?uid=FAZN__20190315_ 6090936\&token=d7b73c72-e971-4a8c-813a-cd9fc086351e\&p._scr=faz-archiv\&p.q=greta +thunbeg+oder+fridays+for+future\&p.source $=\&$ p.max $=10 \& p$. sort $=\& p$. offset $=80 \& p$. ts $=1562070402155 \&$ p.DT_from $=20.08 .2018 \&$ p.DT_to $=01.05 .2019 \&$ p.timeFilterType $=0$ Wir wollen eure Hoffnung nicht. Wir wollen, dass ihr euch uns anschließt Wir schwänzen nicht, wir streiken https://www.faz-biblionet.de/faz-portal/document?uid=RMO

FDA201903145659705\&token=bf369448-e44d-4446-8b7d-0a7b6409d948\&p._scr=fazarchiv\&p.q=greta+thunbeg+oder+fridays+for+future\&p.source $=\& p \cdot \max =10 \&$ p.sort $=$ \&p.offset=90\&p._ts=1562070429830\&p.DT_from $=20.08 .2018 \&$ p.DT_to $=01.05 .2019 \& p$. timeFilterType $=0$

Lehrer-Gewerkschaft lehnt Sanktionen gegen protestierende Schüler ab https://www.faz-biblionet.de/faz-portal/document?uid=FAZN__20190314_ 6088653\&token=1c7b6cee-a5f4-4452-8d21-8b2ec92ae166\&p._scr=faz-archiv\&p.q=greta +thunbeg+oder+fridays+for+future\&p.source $=\&$ p. $m a x=10 \&$ p.sort $=\& p$. offset $=90 \& p$. $\mathrm{t} s=1562070445248 \&$ p.DT from $=20.08 .2018 \&$ p.DT to $=01.05 .2019 \&$ p.timeFilterType $=0$ Greta Thunberg für Friedensnobelpreis vorgeschlagen https://www.faz-biblionet.de/faz-portal/document?uid=FAZN__20190314_ 6088326\&token=86bd4561-1ebd-4d80-bc48-e4194dc4a89e\&p._scr=faz-archiv\&p.q= greta+thunbeg+oder+fridays+for+future\&p.source $=\& p$.max $=10 \& p$. sort=\&p.offset $=$ 90\&p._ts=1562070465977\&p.DT_from=20.08.2018\&p.DT_to=01.05.2019\&p.

timeFilterType $=0$

Klimaschutz im Unterricht Klimadebatte für Jugendliche https://www.faz-biblionet.de/faz-portal/document?uid=RMO FDA201903135654880\&token=93ced663-a066-420b-a160-172c444a4af4\&p. scr=fazarchiv\&p.q=greta+thunbeg+oder+fridays+for+future\&p.source $=\&$ p.max $=10 \&$ p.sort $=$ \&p.offset=90\&p._ts=1562070478578\&p.DT_from $=20.08 .2018 \&$ p.DT_to=01.05.2019\&p. timeFilterType $=0$

Das ist eine Sache für Profis

Schulstreiks mit 25000 Teilnehmern erwartet

https://www.faz-biblionet.de/faz-portal/document?uid=RMO 
Table 3. Continued.

\begin{tabular}{|c|c|c|c|}
\hline No. & Newspaper & Article (title and weblink) & $\begin{array}{c}\text { (Pre- } \\
\text { )selected }\end{array}$ \\
\hline & & $\begin{array}{l}\text { FDA201903095656826\&token=a987d0be-c2ff-49ef-add0-a25d31c71f01\&p._scr=faz- } \\
\text { archiv\&p.q=greta+thunbeg+oder+fridays+for+future\&p.source=\&p.max=10\&p.sort= } \\
\text { \&p.offset=90\&p._ts=1562070503778\&p.DT_from=20.08.2018\&p.DT_to=01.05.2019\&p. } \\
\text { timeFilterType=0 }\end{array}$ & \\
\hline 129 & & Schlotternde Beine und ein Ausflug in den Chemieunterricht & \\
\hline 130 & & $\begin{array}{l}\text { Kolossale Jugend } \\
\text { https://www.faz-biblionet.de/faz-portal/document?uid=FAZN_20190309_ } \\
\text { 6080226\&token=c72d543b-f88f-4384-add1-f9cf690eabb0\&p._scr=faz-archiv\&p.q= } \\
\text { kolossale+jugend\&p.source=\&p.max=10\&p.sort=\&p.offset=0\&p._ts=1562070675211\&p. } \\
\text { DT_from=20.08.2018\&p.DT_to=01.05.2019\&p.timeFilterType=0 }\end{array}$ & $\mathrm{x}$ \\
\hline 131 & & $\begin{array}{l}\text { Ob Schulstreiks für das Klima in Ordnung sind } \\
\text { https://www.faz-biblionet.de/faz-portal/document?uid=FAZN_20190308__ } \\
\text { 6076847\&token=c5382777-bc3e-4f9e-bea5-490d6bbfd7b7\&p._scr=faz-archiv\&p.q= } \\
\text { greta+thunbeg+oder+fridays+for+future\&p.source=\&p.max=10\&p.sort=\&p.offset= } \\
\text { 100\&p._ts=1562070526364\&p.DT_from=20.08.2018\&p.DT_to=01.05.2019\&p. } \\
\text { timeFilterType=0 }\end{array}$ & $\mathrm{x}$ \\
\hline 132 & & $\begin{array}{l}\text { Steinmeier lobt Schülerdemos für Klimaschutz } \\
\text { https://www.faz-biblionet.de/faz-portal/document?uid=FAZN_20190308_ } \\
\text { 6078896\&token=5618635e-ab8d-4e73-8de3-adc7f9dde41c\&p._scr=faz-archiv\&p.q= } \\
\text { greta+thunbeg+oder+fridays+for+future\&p.source=\&p.max=10\&p.sort=\&p.offset= } \\
\text { 100\&p._ts=1562070539766\&p.DT_from=20.08.2018\&p.DT_to=01.05.2019\&p. } \\
\text { timeFilterType=0 }\end{array}$ & $\mathrm{x}$ \\
\hline 133 & & $\begin{array}{l}\text { Hessens Schüler rufen zu landesweiter Klima-Demo auf } \\
\text { https://www.faz-biblionet.de/faz-portal/document?uid=FAZN_20190308__ } \\
\text { 6078619\&token=d05ca162-7e7b-42ae-8d08-e91eeea8fbb7\&p._scr=faz-archiv\&p.q= } \\
\text { greta+thunbeg+oder+fridays+for+future\&p.source=\&p.max=10\&p.sort=\&p.offset= } \\
\text { 100\&p._ts=1562070555467\&p.DT_from=20.08.2018\&p.DT_to=01.05.2019\&p. } \\
\text { timeFilterType=0 }\end{array}$ & $\mathrm{x}$ \\
\hline 134 & & Frauen, die mehr wollen & \\
\hline 135 & & Hasskommentare als gutes Omen & \\
\hline 136 & & $\begin{array}{l}\text { In Panik mit Greta } \\
\text { https://www.faz-biblionet.de/faz-portal/document?uid=FAZ } \\
\text { FD1201903025648531\&token=8c2e7950-15fe-4caa-abcf-6829dc8c6f1b\&p._scr=faz- } \\
\text { archiv\&p.q=in+panik+mit+greta\&p.source=\&p.max=10\&p.sort=\&p.offset=10\&p._ts= } \\
\text { 1562070652944\&p.DT_from=20.08.2018\&p.DT_to=01.05.2019\&p.timeFilterType=0 }\end{array}$ & $\mathrm{x}$ \\
\hline 137 & & $\begin{array}{l}\text { Die Jugend lehnt sich auf } \\
\text { https://www.faz-biblionet.de/faz-portal/document?uid=FAZN_20190302__ } \\
\text { 6068905\&token=4bf7b37d-824c-4aef-a90d-42067b89f3d4\&p._scr=faz-archiv\&p.q=die } \\
\text { +jugend+lehnt+sich+auf\&p.source=\&p.max=10\&p.sort=\&p.offset=10\&p._ts= } \\
\text { 1562070845289\&p.DT_from=20.08.2018\&p.DT_to=01.05.2019\&p.timeFilterType=0 }\end{array}$ & $\mathrm{x}$ \\
\hline 138 & & $\begin{array}{l}\text { Merkel lobt Schülerdemos } \\
\text { https://www.faz-biblionet.de/faz-portal/document?uid=FAZN_20190302_- } \\
\text { 6068538\&token=cf60b7ba-9409-4f0d-b571-18302a6867b2\&p._scr=faz-archiv\&p.q= } \\
\text { merkel+lobt+schülerdemos\&p.source=\&p.max=10\&p.sort=\&p.offset=0\&p._ts= } \\
\text { 1562070877895\&p.DT_from=20.08.2018\&p.DT_to=01.05.2019\&p.timeFilterType=0 }\end{array}$ & $\mathrm{x}$ \\
\hline 139 & & $\begin{array}{l}\text { Karliczek lehnt Demonstrieren in der Schulzeit ab } \\
\text { https://www.faz-biblionet.de/faz-portal/document?uid=FAZ } \\
\text { FD3201903015649758\&token=61efc005-8edc-4eda-aa56-24281a60acf6\&p._scr=faz- } \\
\text { archiv\&p.q=karliczek+lehnt+ab\&p.source=\&p.max=10\&p.sort=\&p.offset=0\&p._ts= } \\
\text { 1562070904050\&p.DT_from=20.08.2018\&p.DT_to=01.05.2019\&p.timeFilterType=0 }\end{array}$ & $x$ \\
\hline 140 & & $\begin{array}{l}\text { Applaus für Greta, Buhrufe für Merkel } \\
\text { https://www.faz-biblionet.de/faz-portal/document?uid=FAZN_20190301_- } \\
\text { 6066961\&token=221a075e-2c9d-45f5-a204-eb35e7d8a9fa\&p._scr=faz-archiv\&p.q=greta } \\
\text { +thunbeg+oder+fridays+for+future\&p.source=\&p.max=10\&p.sort=\&p.offset=100\&p.- } \\
\text { ts=1562070589599\&p.DT_from=20.08.2018\&p.DT_to=01.05.2019\&p.timeFilterType=0 }\end{array}$ & $x$ \\
\hline 141 & & Make earth cool again! & \\
\hline 142 & & $\begin{array}{l}\text { Das rechtfertigt nicht das Schulschwänzen } \\
\text { https://www.faz-biblionet.de/faz-portal/document?uid=FAZN_20190228_ } \\
\text { 6065462\&token=3c30908c-cba5-4b96-8be5-a23692599fad\&p._scr=faz-archiv\&p.q=\% } \\
\text { 22das+rechtfertigt+nicht+das+schulschwänzen\%22\&p.source=\&p.max=10\&p.sort=\&p. }\end{array}$ & $\mathrm{x}$ \\
\hline
\end{tabular}


Table 3. Continued.

\begin{tabular}{lcc}
\hline & & (Pre- \\
No. Newspaper & Article (title and weblink) & )selected
\end{tabular}

offset=0\&p._ts=1562070971260\&p.DT_from=20.08.2018\&p.DT_to=01.05.2019\&p.

timeFilterType $=0$ sort=\&p.offset=0\&p._ts=1562070934651\&p.DT_from=20.08.2018\&p.DT_to=01.05. 2019\&p.timeFilterType $=0$

https://www.faz-biblionet.de/faz-portal/document?uid=FAZ

FD1201902255646199\&token=b3e09fbc-fb42-4a7f-bcc1-a04a2429c0d3\&p._scr=fazarchiv\&p.q=wer+hat+angst+vor+greta\&p.source=\&p.max=10\&p.sort=\&p.offset=0\&p. ts $=1562070997070 \&$ p.DT_from $=20.08 .2018 \& p . D T \_t o=01.05 .2019 \&$ p.timeFilterType $=0$ archiv\&p.q=die+greta+thunberg+aus+hattersheim\&p.source=\&p.max=10\&p.sort=\&p. offset=0\&p._ts=1562071017789\&p.DT_from=20.08.2018\&p.DT_to=01.05.2019\&p. timeFilterType $=0$ archiv\&p.q=protest+am+freitag+fridays+for+future\&p.source=\&p.max=10\&p.sort=\&p. offset=10\&p._ts=1562071062574\&p.DT_from=20.08.2018\&p.DT_to=01.05.2019\&p. timeFilterType $=0$

Von Autisten mehr Sachlichkeit lernen

Friss Tofu, du Würstchen

Für Tausende Schüler ist wieder ein freier Friday

https://www.faz-biblionet.de/faz-portal/document?uid=FAZ FD3201902165640216\&token=99cbcf3e-3aee-4236-9ad4-6ae2633ebe87\&p._scr=fazarchiv\&p.q=für+tausende+schüler+ist+wieder+ein+freier+friday\&p.source=\&p.max= $10 \&$ p.sort=\&p.offset=0\&p._ts=1562071158460\&p.DT_from=20.08.2018\&p.DT_to=01.05. 2019\&p.timeFilterType $=0$ archiv\&p.q=\%22wir+streiken $\% 2 C+$ bis + ihr + handelt $\% 22 \&$ p.source $=\& p . \max =10 \& p$. sort $=$ \&p.offset=0\&p._ts=1562071187734\&p.DT_from=20.08.2018\&p.DT_to=01.05.2019\&p. timeFilterType $=0$

Von Demokratie und Demonstrationen

Die Welt, wie sie ihr gefällt https://www.faz-biblionet.de/faz-portal/document?uid=FAZN__20190211_ 6036155\&token=a75843b5-f378-4a85-9de4-58c35467d1eb\&p._scr=faz-archiv\&p.q=\% $22 \mathrm{die}+$ welt $\% 2 \mathrm{C}+$ wie+sie+ihr+gefällt\%22+greta+thunberg\&p.source=FAZN\&p.max $=$ $10 \&$ p.sort $=\&$ p.offset $=0 \&$ p._ts $=1562071446534 \&$ p.DT_from $=01.01 .2019 \& p . D T \_t o=01.03$. 2019\&p.timeFilterType $=0$

Ziemiak spottet über Klimaaktivistin Thunberg https://www.faz-biblionet.de/faz-portal/document?uid=FAZN__20190210_ 6033857\&token=13a186a9-ae83-4d2d-be93-9156a510c619\&p._scr=faz-archiv\&p.q= ziemiak+spottet+über+klimaaktivistin+thunberg\&p.source=FAZN\&p.max=10\&p.sort= \&p.offset=0\&p._ts=1562071472600\&p.DT_from $=01.01 .2019 \& p . D T \_t o=01.03 .2019 \& p$. timeFilterType $=0$ 
Table 3. Continued.

\begin{tabular}{llc}
\hline No. Newspaper & (Pre- \\
Article (title and weblink) & )selected \\
\hline
\end{tabular}

Klimaschutz entbindet nicht von Schulpflicht

https://www.faz-biblionet.de/faz-portal/document?uid=RMO

FDA201902085632599\&token=828da19e-ccf7-4bf5-bd82-d9636a88050c\&p._scr=faz-

archiv\&p.q=\%22klimaschutz+entbindet+nicht+von+Schulpflicht\%22\&p.source=\&p.

$\max =10 \& p . s o r t=\& p . o f f s e t=0 \& p . \_t s=1562072235521 \&$ p.DT_from $=01.11 .1949 \& p$.

timeFilterType $=0$

$158 \quad$ Marburger OB schickt Einladung an Klimaaktivistin

https://www.faz-biblionet.de/faz-portal/document?uid=FAZN__20190208_

6031452\&token=7e04d48d-3e10-457b-b6c0-dee2f8cfe740\&p._scr=faz-archiv\&p.q=

Marburger+OB+schickt+Einladung+an+Klimaaktivistin\&p.source=\&p.max=10\&p.sort=

\&p.offset=0\&p._ts=1562071942952\&p.DT_from=01.11.1949\&p.timeFilterType=0

AfD kritisiert Schüler-Demos

https://www.faz-biblionet.de/faz-portal/document?uid=FAZN__20190207_

6029152\&token=f97a048a-bc43-4b20-be98-b789dc844c99\&p._scr=faz-archiv\&p.q=AfD

+ kritisiert+Schüler-Demos\&p.source=\&p.max $=10 \&$ p.sort $=\& p$.offset $=0 \& p .+t s=$

1562071966535\&p.DT_from=01.11.1949\&p.timeFilterType $=0$

Die Selbstgefälligkeit des Umwelt-Weltmeisters

Für Eisbären und für Holland

Schüler protestieren gegen Klimapolitik

https://www.faz-biblionet.de/faz-portal/document?uid=RMO

FDA201902025627741\&token=e388bd9c-6fb8-42a4-af44-de63ac4ce05c\&p._scr=fazarchiv\&p.q=schüler+protestieren+gegen+klimapolitik\&p.source=\&p.max=10\&p.sort= \&p.offset=0\&p._ts=1562071992473\&p.DT_from=01.11.1949\&p.timeFilterType $=0$

Auf der Straße statt im Klassenzimmer

https://www.faz-biblionet.de/faz-portal/document?uid=RMO

FDA201902015624507\&token=d0a586ec-56b3-49bf-88b8-3be27863ebf1\&p._scr=faz-

archiv\&p.q=protest $+a m+$ freitag + fridays + for + future $\&$ p. source $=\& p . \max =10 \& p . s o r t=\& p$. offset=10\&p._ts=1562071089943\&p.DT_from=20.08.2018\&p.DT_to=01.05.2019\&p. timeFilterType $=0$

Protest am Zeugnistag

https://www.faz-biblionet.de/faz-portal/document?uid=RMO

FDA201902015626547\&token=9702a4f3-f84a-43c0-9053-ba0966e70a29\&p._scr=faz-

archiv\&p.q=protest + am+zeugnistag \&p.source $=\&$ p.max $=10 \&$ p.sort $=\&$ p.offset $=0 \& p .+t s=$ 1562072016688\&p.DT_from=01.11.1949\&p.timeFilterType $=0$

Es bringt ja nichts, für eine Zukunft zu lernen, die es nicht gibt https://www.faz-biblionet.de/faz-portal/document?uid=FAZN__20190125_ 6008447\&token=3c9327b8-0f72-47cf-9e68-2a7eaf40674b\&p._scr=faz-archiv\&p.q=\% 22es+bringt+ja+nichts $\% 2$ C+für+eine+zukunft+zu+lernen $\% 2 \mathrm{C}+$ die+es+nicht+gibt $\%$ $22 \&$ p.source $=\&$ p.max $=10 \&$ p.sort $=\&$ p.offset $=0 \&$ p.searchln=TI\&p. $. t s=1562072083861 \& p$. DT_from $=01.11 .1949 \&$.timeFilterType $=0$

Wir fordern einen Kohleausstieg bis spätestens 2030

https://www.faz-biblionet.de/faz-portal/document?uid=FAZN__20190125_

6006356\&token=75e269b2-3310-44df-9ebf-bec79d3ca96c\&p._scr=faz-archiv\&p.q=\% 22 Wir+fordern+einen+Kohleausstieg+bis+spätestens+2030\%22\&p.source=\&p.max= $10 \&$ p.sort=\&p.offset=0\&p._ts=1562072129879\&p.DT_from=01.11.1949\&p.

timeFilterType $=0$

Mit dem Privatjet zur Klimarettung

Schulstreik für das Klima

https://www.faz-biblionet.de/faz-portal/document?uid=RMO

FDA201901195615575\&token=39929938-758b-4647-9f45-865ff84aa15d\&p._scr=fazarchiv\&p.q=schulstreik+für+das+klima\&p.source=\&p.max=10\&p.sort=\&p.offset=30\&p. $\mathrm{ts}=1562072161872 \&$ p.DT_from=01.11.1949\&p.timeFilterType $=0$

Schüler demonstrieren gegen Klimapolitik

https://www.faz-biblionet.de/faz-portal/document?uid=FAZN__20190118_

5995064\&token=3cf3ddc8-43ee-4d5d-abd4-9adfd9c9ad87\&p._scr=faz-archiv\&p.q= schüler+demonstrieren+gegen+klimapolitik\&p.source=\&p.max=10\&p.sort=\&p.offset= 10\&p._ts=1562072204993\&p.DT_from $=01.11 .1949 \&$ p.timeFilterType $=0$ Ihr seid nicht erwachsen genug, die Wahrheit zu sagen 
characterized by an ageist language that is highly deprecatory and negative in character, of the sort that Nuessel (1982) and Gendron et al. (2016) encounter with regard to ageist representations of older adults. The language used in the construction of the youth climate activist as absentee - in terms like 'skipping school', 'wagging school', 'truancy' or 'absenteeism' - puts, in line with Lepianka's findings (2015a, 2015b), a negative spin on the youth climate movement and their climate activism.

\section{The dreamers}

The 'dreamer' is a media image that we find frequently implicitly and indirectly asserted in the FFF coverage (in 21 of 98 articles under study). It is created by reporting on climate activists who are (too) young and did not think well about their claims or by reporting on their claims as being unwarranted and unrealistic. By far mostly in FAZ coverage, the image is created. We observe frequently that claims of the youth climate movement are presented as being unwarranted, as they base upon campaigning capability and agitprop that slide over the diversity of interests (FAZ, 136). This image creation becomes apparent as the addressee is deleted from the sentence here: the young climate activists are not portrayed as an actor. Activists are presented independent of their climate claims, pinpointing at public activism which is misplaced in today's divers claims on climate action. The two excepts of newspapers discourses on pages 11 and 12 exemplify this debate. For example in FAZ (105), this image is extended by coverage that hints at unrealism of activists claims, as the FFF movement is named 'camp of eco-friends' and 'the Greenies', when the FFF got support by Herbert Diess, chairman of the board of Volkswagen AG and VW Passenger Cars brand. The name FFF movement is replaced by a nomination, which refers to the environmentally awareness of young people and deletes the actor from the sentences. The awareness of climate activists on climate action is exaggerated in the final sentence of the article to a utopian dream of Diess never getting into a car again. In addition, both newspapers engage in strategies that explain activists as youngsters who have as yet not thought well about their own claims. As we can observe in the taz example given later, activism is argued to be not very wide-reaching or not permeating activists' life. What becomes apparent in the citations below too, is that youth climate activists are portrayed in reference to stereotypes of young people or 'our children' (unsere Kinder). Here, the journalists make use of known stereotypes that provoke associations of the youth for the reader and subordinate them under adults. It directs focus on a potential flaw of young people: of not knowing enough and not taking things serious due to being a young pupil. Certainly, this gives room to question seriousness and legitimacy of the movement's science-based claims and thereby facilitates judging the FFF movement based on age of the protesters instead of specific founded reasons.

\section{The young heroine}

In regard to our findings on the FFF coverage, it is of certain interest that Greta Thunberg is widely and creatively discussed in the debate. She is typically presented as a young girl who heroically initiated the youth climate movement. This construct of Greta Thunberg as an icon could be found in 71 articles of 98 analysed ones. FAZ and taz both create certain iconicity and heroism of Greta Thunberg by the use of predications and nominations, which present 
her as a role model, as a leader, and as an initiator of youth climate activism. The iconicity of Greta Thunberg is established by FAZ and taz portraying her as an ideal example of greenminded behaviour. However, we also find heroism and iconicity of Greta Thunberg created by a wide use of rhetorical elements. She is compared to prominent and influential personalities, for example to Nobel Laurate in Economic Sciences Elinor Ostrom, Donald Trump, or Abba. Thereby, the iconicity of Greta Thunberg appears in a very abstract and exaggerated manner, which is far from reality and again creating certain distance. Furthermore in taz it is evident that the rhetoric tends to create a messianic coverage and sanctification of her. This is found in citations like 'Gretas mission' (2) 'Greta, Jesus and the climate catastrophe' (11). The youth aspect seems relevant in this image creation too. The young girlhood, that is part of this 'young girl heroism', is mainly created by the newspapers' nominations and predications of her as either young girl or teeny or as a pupil. The categorization of Greta as young girl and the nomination of her as 15-year-old girl shows that she is portrayed from the specific angle of a young girl, in a similar way as Malala Yousafzai has been presented in media discourses. Such coverage facilitates the perception of Greta Thunberg as different, potentially as 'out-group' in terms of her youthfulness. This goes in line with Gendron et al.'s (2016) notion of a medial contribution to out-grouping. The images of the global FFF movement apply to Greta Thunberg as well. We take upon our previous discussion of findings here and do not repeat the paternalistic but inherently ageist language use here. Although presented as an icon, she is not seen as a separate case of the youth climate movement. This gives us room to discuss a certain case, which is that yet, with the imaging of Greta Thunberg, the three images of the FFF movement become personalized.

It is most striking, that in several FAZ articles she is held responsible for the 'absenteeism' of climate activists, as They [the protests] trace back to the Swedish pupil Greta Thunberg who called for skipping school on Fridays to draw attention to urging issues' (FAZ, 162) or Who made it that her comment [Greta Thunberg] on the German coal compromise is not only widespread but she, a 16-year-old Swede, beguiled the CDU general secretary into attacking her in public' (FAZ, 153). In other words, while Greta Thunberg is presented as an icon, her cult of personality comes with descriptions in demeaning and negative language. Our findings corroborate our expectation that paternalist media systems stamp their image of young girl heroism on Greta Thunberg in its youth climate movement discourse. Its media image may sound very positive, yet, its construct of young girl heroism is not abstained from derogatory ageist language and, like its other media images, sheds doubt on youth activism. The personality cult that both FAZ and taz construct in their news coverage, coincide with holding Greta Thunberg responsible for the discursively construction negative perception of the protests against the established environmental governance regime.

\section{The interplay of the four media images}

FAZ (105) title 'climate strike at Volkswagen': 'Do youngsters necessarily have to skip school in order to protest for more climate protection? Who considers that sensible has company whether one wants to or not from the head of the greatest car manufacturer worldwide. Herbert Diess, the chairman of Volkswagen, did not stand out as Germany's supreme climate protector yet - but now he request admission in the camp of the eco-friends. At the end of the week, the head of the car manufacturer spreads an encouragement 
to skipping school. (...). Often, parents were upset because lessons were cancelled due to too little teachers at school. But now pupils should cancel lessons themselves because they have better things to do. Usually everyone is always talking about education of great value. With good reason! Studying serves as a basis for running one's own life, for progress and prosperity - also for fighting climate change. No business representative should ignore that. If absenteeism would be single, one could look over it. But there is no end in sight for school strikes'.

taz (55) title 'Why do we criticize children, who take saving the planet to the streets?': 'Shall they celebrate, shall they skip school, romp about and blare out something, even if it does not cost them anything. It would be at their costs, if they stop going shopping, stop the wasting, if they start to engage seriously, as well in their free time. And that is the next step. A Greta Thunberg, who visits Hamburg on Friday, is a proper fighter already and an icon. She refrains from many. From regular lessons, inconspicuousness, she makes herself vulnerable and she lives by that assaults. All an option. Also for our children'. The derogatory kind of portrayals of youth climate activists as dreamers gives an indication of what prejudices of young people circulate and how such prejudices are used to enforce a status quo. A status quo, in which young people belong in the institution responsible, the school, and they fill the role of subordinated people in the process of growth and education. It is of certain interest that in FAZ coverage, it is more frequently found that ageist images are created in relation to doubts or opposition to the claims of activists, whereas the taz seems positive about climate activism but still inherits ageist doubts on youth activism, its authenticity and 'childishness'. As we observe in the excerpt of FAZ above, the author neither seems convinced by climate activism nor the camp of eco-friend is valued. The created distance to young activists is of certain interest in regard to what environmental linguistics indicate on conservatism and climate skepticism (Jacques 2012; Poortinga et al. 2011; Whitmarsh 2011). The skeptical attitude found in the conservative paper FAZ approves such findings in the German context. Then again, it is striking that FAZ engages in rhetoric that represents Greta as an environmental ideal and icon. It presumes that certain value of environmentalism is perceived but not necessarily promoted. taz coverage cannot be explained by that, but rather it hints at prevailing stereotypes on young people as Lepianka already noted or a deep seated uneasiness as Butler called it in reference to ageing. In the excerpt, taz intentionally argues for climate protest but likewise creates great distance to activists based on age in a strong paternalistic manner. In the vein of Rahmstorf, the FAZ coverage suits well in the category of impact sceptics, who are not convinced by climate changes harm. In the case of taz coverage, a new meaning may be attributed to the term impact sceptics, the impact of youth activism appears to be in question.

At least, the three media images - pupil, absentee and dreamer - appear inter-related. The pupil construct fails to take young people seriously regarding their interests (including their future) and are seen as uneducated, which promotes the idea of claims that are not well-wrought or unrealistic that is associated with the dreamer construct. The image of the absentee directs the focus on breaching the law and being unreliable, which aligns with the notion of judging the claims as illegitimate and activism as not authentic that is associated with the dreamer construct. Differently from the construct of the image of the pupil and the absentee, the media image of the dreamer not only reveals a negative depiction of young people in terms of a lack of schooling or a lack of decency, but in terms of a lack of legitimacy. 


\section{German newspaper coverage in the context of the coal commission}

Since the Paris agreement in 2015, there has been a vibrant public discussion on phasing out coal in Germany. 'Germany, the largest contributor to greenhouse gas emissions in the European Union, has identified the need to phase out coal in order to achieve its climate targets' (Climate Analytics 2018, 1). The main aim of the German FFF movement is to influence the decision of the coal commission on a final date of phasing out coal: it wishes coal to be phased out by 2030 . The FFF takes upon scientific evidence on how to achieve phasing out of coal and to comply with the Paris agreement by that. Climate Analytics (2016, 2017 and 2018), Greenpeace and Fraunhofer (2018), and BUND (2018) argue for a coal phase out until 2030 as consequence for coal-based power generation when aiming at $1.5^{\circ}$ to $2^{\circ}$-degree limit (Climate Analytics 2018). Prognos and ÖkoInstitut find a phasing out of coal until 2035 in order to underscore $2^{\circ}$-degree (WWF Germany 2017).

However, the ambitions of phasing-out of coal by the coal commission are clearly limited. The advice given in their final report on phasing out of coal in Germany is to phase out coal until 2038, if possible until 2035 (BMWi 2019). The advice mismatch leading scientific evidence on Germany's contribution to underscore $2^{\circ}$-degree of global warming by phasing-out of coal as named before. The conditions for phasing-out 2035 are: if energy industrial, employment political and economically feasible requirements are met, the date of negotiations with the operators can preponed earliest in 2035. It reveals that the decision on phasing-out is strictly bound to and oriented on Germany's industrial and economic future instead of its climate goals. Whether early negotiations are possible, shall be checked in 2032 ('Öffnungsklausel'). In the report it is stated that the opening clause includes the evaluation whether the assumptions for ending the coal-based power generation in total is realistic (BMWi 2019,64). This shows a very modest, skeptical tone on phasing-out of coal.

The coal commission seems not convinced to end coal-based power generation. Such doubts seem to underly the discussion and that the term realistic is taken upon here, since the newspaper discourse on FFF (or the dreamers) and Greta Thunberg (or the hero at fault) bore a similar silent reproach. The final report states that the reduction of greenhouse gases emission between 2023 and 2030 is ensued as consistent as possible (BMWi 2019, 63). In regard to compliance with such reduction, neither midterm goals are set nor it clearly specifies the aim of a consistent of reduction. The language used supports the notion of a very reluctant discourse on taking climate action. This finding is supported by the reluctancy in making an argument on Hambacher Forst, a forest in North Rhine-Westphalia that vanishes RWE, one of Germany's largest electric utility company, due to its brown coal mining. The clearing of the Hambacher Forst has presented another issue for climate activists. In the report it is stated that the coal commission feels it desirable that the Hambacher Forest (an ancient forest near the city of Cologne) is preserved $(2019,63)$. Although the commission gives a statement and sees the need to preserve the forest, the rhetoric of the coal commission shows reluctancy in taking climate action in the context of phasing-out of coal. An actual engagement with and commitment to climate protection measures sits on the fence.

Advising a coal phase out later, using reluctant, inherently skeptical language seizes upon coal industrial interests. The coal commission suits well within the category of 
impact sceptics, who a cherry-picking of results with a liberal interpretation that support their position (Rahmstorf 2004, 79). The member list as well as the consultation list of the coal commission is relevant. The coal commission is formed by the German government, not including the opposition. It shows a representation of 21 men and 10 women, who are on average 58 years old. $1 / 3$ of the members ( 9 in total) belong to conservative parties (CDU, CSU) in Germany. This is striking regarding Ford's finding on an exclusive, orthodox environmental governance regime. But also, what Whitmarsh (2011) and Poortinga et al. (2011) uncover on climate sceptics is relevant: climate sceptics are rather man than women, rather older people than younger people and conservative voters. The member list of the coal commission shows that no involvement of the FFF movement was planned (BMWi 2019), which makes, of course, sense given that the movement developed only after August 2018. The civil society platform that was chosen to be heard are citizens and regional representatives of the three largest centres of coal in Germany (Mitteldeutsches Revier, Lausitzer Revier and Rheinisches Revier). It can be assumed that people and regions heard are more interested in job security and regional infrastructure, since local tax income is dependent on coal industry. At this point, we argue that the interests of the coal industry, or impact sceptics (Rahmstorf 2004), is overrepresented in the coal commission as well as by the typical climate sceptics. This becomes apparent in the content as well as the rhetoric of the final report as we outlined earlier. An in-depth study of the socio-demographics of German climate sceptics remains to be done.

\section{Concluding remarks}

Our analysis reveals existing power dynamics over youth climate activists and their interests on phasing-out of coal early (enough) in Germany. In line with CDS, the binary notions of newspaper texts on youth climate activism and context of phasing out coal is abolished. CDA shows that the FAZ and taz newspaper discourse on the youth climate movement has (re)produced this domination over the youth climate movement and their interests by the use of ageist language. Ageist newspaper coverage in a left-wing (taz) as well as in a conservative paper (FAZ) on the youth climate movement reveals their contribution to and maintenance of the exclusive and orthodox global environmental discourse, that predominates over the youth climate movement. CDS indicates that this newspaper discourse cannot be disconnected from the context of the German environmental governance regime. We find similar notions of climate skepticism in the newspaper discourse of FAZ and the discussion of a governmental commission, that spell doom for Germany's coal and hence contribution to global climate action. Both meet in their skeptical discourse on climate change action, as they seem not to attribute much value to it and to believe in its impact. Our most striking findings are not only an ageist language that seems having a paternalistic origin, but also that conservatism bears a relation to climate skeptical discourses, which takes shape in a very reluctant discourse that is not convinced yet to take climate action.

The ageist derogation of the youth climate movement is paternalist. FAZ and taz articles represent the youth climate movement in terms of its identity as pupils; youth climate activism is portrayed as being absent from school; and its claims are narrated as dreams. The media representations of youth climate activists as pupils and absentees strategically divert the discussion of young climate activism - FFF's claims about phasing out coal in 
Germany by 2030 - towards their societal role as not yet fully educated people who are obliged by law to go to school. The prevalence of inequality in Germany's age-related contexts is strongly shown in the newspaper coverage of youth climate activism. The German media's disregard of the interests of the young climate activists on climate action is typical for its ageist domination. In newspaper coverage, young people are portrayed as an inherently different social group, who are to be kept out of public spaces. Although, in line with Lepianka (2015b), we expected that this portrayal is key to 'othering' youngsters, it is unexpected that this othering includes an image of illegitimate youth climate activism and a structural underestimation of young activists' abilities and capabilities of knowing and exerting their climate interests. Media propensity to criminalize youth remains obvious. Yet, this underestimation is typical for paternalism. The exertion of paternalism aligns with the literature on the exposure of young people, including Greta Thunberg, to paternalism in the public setting (De Valk et al. 2016; Sheleff 1984). In the exposure of young people to paternalist domination, essentialist relations between young and old are reinforced, with young people being portrayed as lacking experience and being unable to see to their own interests as children. It is a German paternalism - with FAZ and taz discursively aligning with the hegemonic environmental governance discourse that is, amongst other actors, articulated by the German coal commission - that fails to emphasize required measures to phase out coal in time, not thinking to include political youth who should not fight for their ecological future but should go to school instead.

Greta Thunberg - depicted by the OPEC cartel as the 'greatest threat' to the fossil fuel industry (The Guardian 2019) - is in German newspapers typically portrayed as the young pioneer and ideal of environmentalism these days. It is noticeable that journalists' rhetoric tends to sanctify her behaviour. She is exposed to ageism in the newspaper discourse too, but it is surprising that the images with its ageist expressions that are created to other the movement in general, are personalized and directed on her as problematic and disorderly. The alleged problem is mainly the activism of 'pupils' in general, the 'absenteeism' of the protesters, and their ideas for phasing out coal. The ambiguity between the newspaper coverage of Greta Thunberg as the hero and role model and the portrayal of her person as partly at fault stands out. This discourse personalizes the debate on youth climate activism and environmental governance in Germany on her as single individuum. By doing so, the depiction of Greta Thunberg recedes the reader of her person, creating a cult of personality, which certainly makes her an instrument of discussion.

The youth climate movement do not collapse in the public discourse on climate politics but continues action. This contradicts scientific literature on (youth) climate activism (Burciaga and Martinez 2017; O'Brien, Selboe and Hayward 2018). Access to and engagement with democratic institutions is required in order to form 'alternatives that in the long run can threaten vested interests and the status quo in unconventional ways' (O'Brien et al. 2018; also see Gencoglu and Yarkin 2019). The ongoing protests of the FridaysForFuture movement worldwide and Greta Thunberg hints at a strong environmentally awareness of young people today. What our paper hints at, is that international climate goals are far from being achieved and the youth climate movement still needs great support, to resist ageist hegemonic representations of young people in the context of a biased environmental governance regime. 


\section{Acknowledgements}

We thank the editor of this journal, Shi-Xu, for giving us the opportunity to share this study with the readers as well as we thank the peer referees, whose comments were very much helpful.

\section{Disclosure statement}

No potential conflict of interest was reported by the author(s).

\section{Notes on contributors}

Marinus Ossewaarde is an associate professor in sociology of governance at the University of Twente, Netherlands. His most recent book is Theorizing European Societies (Basingstoke: Palgrave Macmillan, 2013). He has published a variety of articles on technology and society topics, including multiculturalism, Eurocentrism, discourses, resistance, domination, ideology, cosmopolitanism, memory, identity, bureaucracy, publics, digital commons and dialectic in journals like Organization Studies, Organization, Futures, Current Sociology, Critical Sociology, European Journal of Social Theory, European Societies, Sociology, and The European Legacy.

Zoe Bergmann is a junior researcher at the University of Twente, Netherlands. Her main research interests include political and media discourse and domination.

\section{References}

BUND. 2018. BUND-Abschaltplan für AKW und Kohlekraftwerke. https://www.bund.net/fileadmin/ user_upload_bund/publikationen/kohle/kohle_bund_abschaltplan_kohle_atom.pdf.

Burciaga, E.M., and L.M. Martinez. 2017. How do political contexts shape undocumented youth movements? Evidence from three immigrant destinations. Mobilization: An International Quarterly 22, no. 4: 451-471. doi:10.17813/1086-671x-22-4-451.

Butler, R. N. 1969, June 11. Age-ism: another form of bigotry. Punch. https://pdfs.semanticscholar.org/ c31c/60684ed6a62e5142700842f4adc34435189c.pdf.

Climate Analytics. 2016. Implications of the Paris agreement for coal use in the power sector. https:// climateanalytics.org/media/climateanalytics-coalreport_nov2016_1.pdf.

Climate Analytics. 2017. A stress test for coal in Europe under the Paris agreement. https:// climateanalytics.org/media/eu_coal_stress_test_report_2017.pdf.

Climate Analytics. 2018. Science based coal phase-out pathway for Germany in line with the Paris Agreement $1.5^{\circ} \mathrm{C}$ warming limit: Opportunities and benefits of an accelerated energy transition. https://climateanalytics.org/media/germany_coalphaseout_report_climateanalytics_final.pdf.

Coupland, N., and J. Coupland. 1993. Discourses of ageism and anti-ageism. Journal of Aging Studies 7, no. 3: 279-301. doi:10.1016/0890-4065(93)90016-D.

De Valk, S., C. Kuiper, G.H.P. Van der Helm, A.J.J.A. Maas, and G.J.J.M. Stams. 2016. Repression in residential youth care: A scoping review. Adolescent Research Review 1, no. 3: 195-216. doi:10.1007/ s40894-016-0029-9.

Edström, M. 2018. Visibility patterns of gendered ageism in the media buzz: A study of the representation of gender and age over three decades. Feminist Media Studies 18, no. 1: 77-93. doi:10.1080/ 14680777.2018.1409989.

FAZ.NET. 2018, July 2020. Digitale Reichweite der FAZ stark gestiegen. Frankfurter Allgemeine Zeitung. https://www.faz.net/aktuell/wirtschaft/digitale-reichweite-der-faz-stark-gestiegen-15699377.html.

Federal Ministry for Economic Affairs and Energy [BMWi]. 2019. Kommission "Wachstum, Strukturwandel und Beschäftigung". Abschlussbericht. https://www.bmwi.de/Redaktion/DE/ Downloads/A/abschlussbericht-kommission-wachstum-strukturwandel-und-beschaeftigung.pdf? _blob=publicationFile. 
Ford, L.H. 2003. Challenging global environmental governance: social movement agency and global civil society. Global Environmental Politics 3, no. 2: 120-134. doi:10.1162/152638003322 068254.

Fraser, S.A., V. Kenyon, M. Lagacé, W. Wittich, and K.E. Southall. 2016. Stereotypes associated with age-related conditions and assistive device use in Canadian media. The Gerontologist 56, no. 6: 1023-1032.

Fridays For Future Germany [FFF Germany]. 2019. Unsere Forderungen an die Politik. https:// fridaysforfuture.de/forderungen/.

Gencoglu, F., and D. Yarkin. 2019. The student movement in Turkey: A case study of the relationship between (re)politicization and democratization. Journal of Youth Studies 22, no. 5: 658-677. doi:10. 1080/13676261.2018.1535170.

Gendron, T.L., E.A. Welleford, J. Inker, and J.T. White. 2016. The language of ageism: Why we need to use words carefully. The Gerontologist 56, no. 6: 997-1006.

Greenpeace and Fraunhofer. 2018. 2030 kohlefrei. Wie eine beschleunigte Energiewende Deutschlands Beitrag zum Pariser Klimaschutzabkommen sicherstellt. https://www.greenpeace. de/sites/www.greenpeace.de/files/publications/2030_kohlefrei_fraunhofer_iee_greenpeace.pdf.

The Guardian. 2019, July 5. 'Biggest compliment yet': Greta Thunberg welcomes oil chief's 'greatest threat' label. The Guardian.

Haynes, S. 2019, May 24. Students from 1,600 Cities just walked out of school to protest climate change. It could be Greta Thunberg's biggest strike yet. Time USA. https://time.com/5595365/ global-climate-strikes-greta-thunberg/.

Jacques, P.J. 2012. A general theory of climate denial. Global Environmental Politics 12, no. 2: 9-17.

Kaiser, J., and M. Rhomberg. 2016. Questioning the doubt: climate skepticism in German newspaper reporting on COP17. Environmental Communication 10, no. 5: 556-574.

Khurshid, A., and B. Pitts. 2019. Malala: the story of a Muslim girl and a Muslim nation. Discourse: Studies in the Cultural Politics of Education 40, no. 3: 424-435. doi:10.1080/01596306. 2017.1346587.

Kroon, A.C., D. Trilling, M. Van Selm, and R. Vliegenthart. 2019. Biased media? How news content influences age discrimination claims. European Journal of Ageing 16: 1.

Lamb, E.C. 2013. Power and resistance: new methods for analysis across genres in critical discourse analysis. Discourse \& Society 24, no. 3: 334-360. doi:10.1177/0957926512472041.

Lepianka, D. 2015a. The representation of youth in the Dutch news media. Young 23, no. 4: 277-292. doi:10.1177/1103308815596881.

Lepianka, D. 2015b. How similar, how different? on Dutch media depictions of older and younger people. Ageing and Society 35, no. 5: 1095-1113. doi:10.1017/S0144686X14000142.

Levinsen, K., and C. Wien. 2011. Changing media representations of youth in the news - A content analysis of Danish newspapers 1953-2003. Journal of Youth Studies 14, no. 7: 837-851. doi:10. 1080/13676261.2011.607434.

Lloyd-Sherlock, P.G., S. Ebrahim, M. McKee, and M.J. Prince. 2016. Institutional ageism in global health policy. BMJ (Clinical Research edition) 354: i4514. doi:10.1136/bmj.i4514.

Moris, M., and M. Loopmans. 2019. De-marginalizing youngsters in public space: Critical youth workers and local municipalities in the struggle over public space in Belgium. Journal of Youth Studies 22, no. 5: 694-710. doi:10.1080/13676261.2018.1535172.

Nuessel, F.H. 1982. The language of ageism. The Gerontologist 22, no. 3: 273-276.

O'Brien, K., E. Selboe, and B.M. Hayward. 2018. Exploring youth activism on climate change: Dutiful, disruptive, and dangerous dissent. Ecology and Society 23: 3. doi:10.5751/ES-10287-230342.

Olesen, T. 2016. Malala and the politics of global iconicity. The British Journal of Sociology 67, no. 2: 307-327. doi:10.1111/1468-4446.12195.

Poortinga, W., A. Spence, L. Whitmarsh, S. Capstick, and N.F. Pidgeon. 2011. Uncertain climate: An investigation into public scepticism about anthropogenic climate change. Global Environmental Change 21, no. 3: 1015-1024.

Pritchard-Jones, L. 2017. Ageism and autonomy in health care: Explorations through a relational lens. Health Care Analysis: HCA: Journal of Health Philosophy and Policy 25, no. 1: 72-89. doi:10.1007/ s10728-014-0288-1. 
Qazi, H., and S. Shah. 2018. Identity constructions through media discourses. Journalism Studies 19, no. 11: 1597-1612. doi:10.1080/1461670X.2017.1284574.

Rahmstorf, S. 2004. The climate sceptics. Weather Catastrophes and Climate Change, 76-83.

Rhomberg, M., J. Kaiser, D. Lichtenstein, and B. Faehnrich. 2018. Rise and fall of a global symbol: Frames of the 2degree limit in German News Media 2009-2014. Meteorologische Zeitschrift 27 no. 2: 149-160.

Sheleff, L.S. 1984. Paternalism and the young. Children and Youth Services Review 6, no. 4: 267-283. doi:10.1016/0190-7409(84)90047-1.

Shi-Xu. 2016. Cultural discourse studies through the journal of multicultural discourses: 10 years on.

Stegemann, L., and M. Ossewaarde. 2018. A sustainable myth: A neo-Gramscian perspective on the populist and post-truth tendencies of the European green growth discourse. Energy Research \& Social Science 43: 25-32.

taz.de. 2017. Zahlen und Fakten: Wir über uns. die tageszeitung. https://taz.de/Zahlen-und-Fakten/! $106557 /$.

Van Dijk, T.A. 1993. Principles of critical discourse analysis. Discourse \& Society 4, no. 2: 249-283.

Welch, M., E. Price, and N. Yankey. 2002. Moral panic over youth violence: Wilding and the manufacture of menace in the media. Youth \& Society 34, no. 1: 3-30.

Westman, J.C. 1991. Juvenile ageism: Unrecognized prejudice and discrimination against the young. Child Psychiatry Human Development 21, no. 4: 237-256.

Whitmarsh, L. 2011. Scepticism and uncertainty about climate change: Dimensions, determinants and change over time. Global Environmental Change 21, no. 2: 690-700.

WISO databank. 2019a. Ihr Suchergebnis (13.355). https://www.wiso-net.de/dosearch?_searchOnlyln AbstractField=\&_searchOnlylnTitleField=\&explicitSearch=true\&q=greta+thunberg+oder+fridays + for+future+\&dbShortcut=\%3A3\%3AALLEQUELLEN-166_\%3A3\%3APRESSEDTL\&searchMask= $7168 \&$ TI\%2CUT\%2CDZ\%2CBT\%2COT\%2CSL=\&KO=\&MM\%2COW\%2CUF\%2CMF\%2CAO\%2CTP\% 2CVM\%2CNN\%2CNJ\%2CKV\%2CZ2=\&CO\%2CC2\%2CTA\%2CKA\%2CVA\%2CZ1=\&CT\%2CZ4\% 2CKW=\&BR\%2CGW\%2CN1\%2CN2\%2CNC\%2CND\%2CSC\%2CWZ\%2CZ5\%2CAI\%2CBC\%2CKN\% 2CTN\%2CVN\%2CK0\%2CB4\%2CNW=\&Z3\%2CCN\%2CCE\%2CKC\%2CTC\%2CVC=\&timeFilterType= on\&DT_from $=20.08 .2018 \& D T \_t o=01.05 .2019 \& x=87 \& y=22$.

WISO databank. 2019b. Ihr Suchergebnis (16.222). https://www.wiso-net.de/dosearch?_searchOnly InAbstractField=\&_searchOnlyInTitleField=\&explicitSearch=true\&q=greta+thunberg+oder+friday $\mathrm{s}+$ for+future\&dbShortcut=\%3A3\%3AALLEQUELLEN-165_\%3A3\%3APRESSE\&searchMask= 7012\&TI\%2CUT\%2CDZ\%2CBT\%2COT\%2CSL=\&KO_and_1=\&MM\%2COW\%2CUF\%2CMF\%2CAO\% 2CTP\%2CVM\%2CNN\%2CNJ\%2CKV\%2CZ2=\&CO\%2CC2\%2CTA\%2CKA\%2CVA\%2CZ1=\&CT\% $2 \mathrm{CZ} 4 \% 2 \mathrm{CKW}=\& \mathrm{BR} \% 2 \mathrm{CGW} \% 2 \mathrm{CN} 1 \% 2 \mathrm{CN} 2 \% 2 \mathrm{CNC} \% 2 \mathrm{CND} \% 2 \mathrm{CSC} \% 2 \mathrm{CWZ} \% 2 \mathrm{CZ} \% 2 \mathrm{2CAI} \% 2 \mathrm{CBC} \%$ 2CKN\%2CTN\%2CVN\%2CK0\%2CB4\%2CNW=\&Z3\%2CCN\%2CCE\%2CKC\%2CTC\%2CVC $=\& S O \% 2 C Z K$ $\% 2 C Z T=\&$ timeFilterType=on\&DT_from $=20.08 .2018 \& D T \_t o=01.05 .2019 \& x=62 \& y=7$.

Wodak, R. 2012. Language, power and identity. Language Teaching 45, no. 2: 215-233.

WWF Germany. 2017. Zukunft Stromsystem. Kohleausstieg 2035. Vom Ziel her denken. https://www. prognos.com/uploads/tx_atwpubdb/20170123_Prognos_StudieStromsystemKohleausstieg2035_ Final_Kor_Web.pdf. 